\begin{tabular}{c} 
International Journal of Engineering \& Technology, 7 (3.10) (2018) 184-186 \\
International Journal of Engineering \& Technology \\
SPC \\
Website: www.sciencepubco.com/index.php/IJET \\
Research paper \\
\hline
\end{tabular}

\title{
Categorization of Vehicle and Motion Analysis Using Vehicle Features
}

\author{
Ms.S.Vanithamai ${ }^{1 *}$, Dr.S.Purushothaman ${ }^{2}$ \\ ${ }^{1}$ Assistant Professor, Master of Computer Applications, M.Kumarasamy College of Engineering \\ ${ }^{2}$ Professor \\ "Corresponding author E-mail: svanithasubu@gmail.com
}

\begin{abstract}
This research work can identify the vehicle and classify the vehicle using the vehicle features such as shape, color etc., The parameters extracted from the vehicle classification are based on movement of the vehicle are classified as static, movement variation in the successive video frames are used to identify the hazardness of the vehicle. Digital Image processing techniques are used in the object detection.
\end{abstract}

Keywords: Vehicle detection, Tracking, Motion Analysi

\section{Introduction}

This research work focuses in applying image processing concepts for detecting and tracking vehicles. Videos collected from different conditions in which vehicles were moving are used for analysis. Videos have been used as it is non-intrusive process and can be analyzed offline.

Vehicle should follow traffic rules and move inside a lane for implementing the proposed algorithms. If the vehicle is moving inside lane, false rejection of an actual vehicle or false identification of an object which is not a vehicle can be minimized. The following steps show how to identify a vehicle and direction in which vehicle is moving.

Step 1:Identify if it is a vehicle. Discard other objects.

Step 2:Verify if the vehicle is moving.

Step 3:For each vehicle identified in a frame, a vector consisting of \{width, height, segmented object, average intensity value, road brightness (dark/light/bright)\} is created. This step is required for tracking the vehicle in the subsequent frames.

Step 4: Find the change in the vehicle size, and the direction of vehicle can be obtained. If the vehicle size grows, it indicates that the vehicle is coming towards the camera.

The next position of vehicle-1 to vehicle-N are obtained in the frames $(n+1)$ s by finding the $x, y$ locations of the vehicles- 1 to $\mathrm{N}$ as mentioned below:.

$\mathrm{V}_{1}(\mathrm{n}+1)_{\mathrm{x}, \mathrm{y}-} \mathrm{V}_{1}(\mathrm{n})_{\mathrm{x}, \mathrm{y}}$

$\mathrm{V}_{2}(\mathrm{n}+1)_{\mathrm{x}, \mathrm{y}}-\mathrm{V}_{2}(\mathrm{n})_{\mathrm{x}, \mathrm{y}}$

$\mathrm{V}_{\mathrm{N}}(\mathrm{n}+1)_{\mathrm{x}, \mathrm{y}-} \mathrm{V}_{\mathrm{N}}(\mathrm{n})_{\mathrm{x}, \mathrm{y}}$

A vehicle can miss in some of the frames due to the following:

1. Overlap with other vehicles inline.

2. Has taken a left or right turn.

\section{Categories of Vehicle Detection}

There is an increase in traffic flow due to development of vehicle technologies and transport systems. Improved moni- are detected and tracked in all weather conditions . Detecting the movement of the vehicle in video sequence is difficult because of illumination condition background images, and occlusion, unexpected object motion, change in form of the object pattern, non-rigid structures of object. The problem is to evolve new techniques to

1. Tripline:User defines the detection zone in the road way. Sensors are used to estimate the vehicle speed that considers the time it takes an identified vehicle to traverse a detection zone of known length. When the vehicle crosses one of the user identified zones, changes in the pixel are monitored.

2. Closed-loop tracking : It is applicable for the longer roadway sections. Vehicles are indentified in a particular track as multiple selections. Validation can be performed and that particular vehicle has been taken into account for counting and speed of the vehicle is calculated using tracking algorithm. It is useful to alert the driver in a critical situation and to avoid the accident.

3. Data association tracking : This techniques connects the unique pixels to detect and track a specific vehicle or group of vehicles. The vehicles are assumed that it pass through the field of view of the camera. These unique connected pixels are tracked for every frame to give up tracking data for the selected vehicle

\section{Data Collection}

Video database has been created by shooting video in hand DIGICAM. The videos are transferred to laptop. OSS decompiler has been used to extract the frames of the video. The video has been taken in the day time, cloudy time, late evening and at night. All the information are processed using MATLAB R2011a. Using MATLAB we can get the solution faster than traditional programming languages. The following frames show distinctly the road, track lines and vehicles when viewed inclined, straight and curved. Fig 1 presents sample frames of video taken by fixing camera in a car 

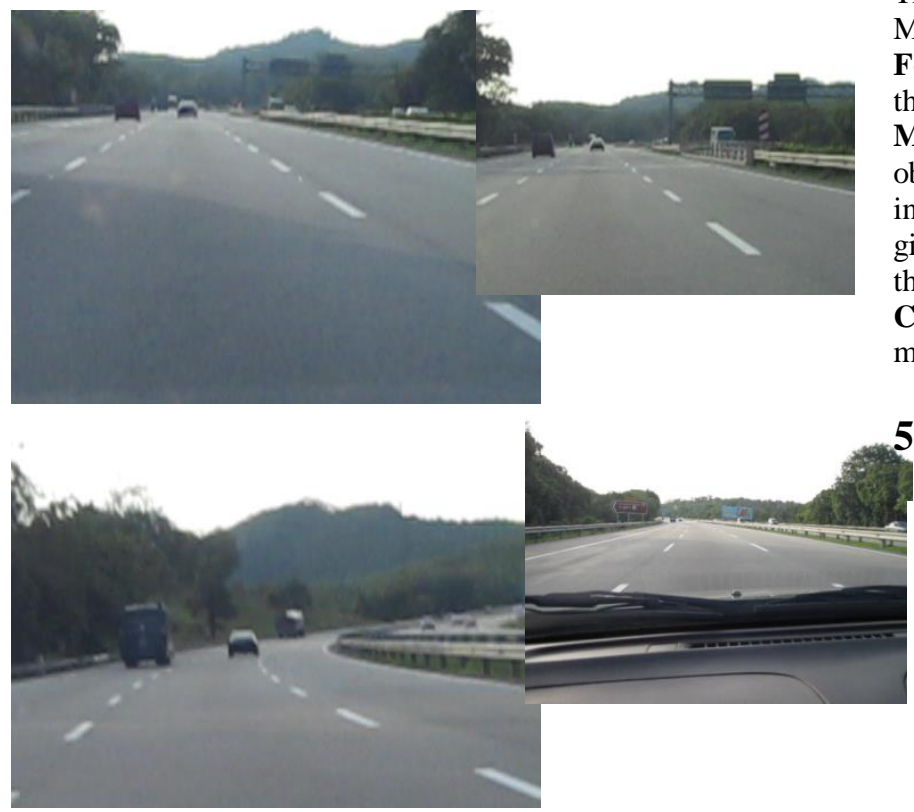

Fig. 1 : Sample frames

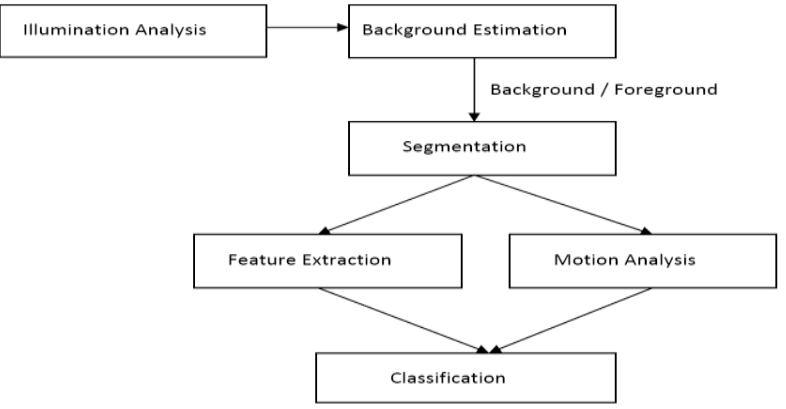

Fig. 2 : Classification by proposed algorithms

\section{Procedure for Object Classification}

In the Fig 2, each single rigid and moving/static object is tracked. Each object is enclosed with rectangle, with the following properties: $\mathrm{X}$ and $\mathrm{Y}$ coordinates of the top left and bottom right of the rectangle, $\mathrm{X}$ and $\mathrm{Y}$ coordinates of the contour points of the tracked objects. Motion features extracted will be current speed, acceleration value, deceleration value, change in size, change in velocity. The movement of the vehicle is determined based on the previous frames to current frame and are listed as follows: static to static, static to moving, moving to moving, moving to static, sudden stop, sudden speed.

Illumination Analysis: Basic input for this objective is to capture a video sequence of frames. Raw image is captured by the video camera using optical sensors. Determining the perfect illumination is important for the quality of the captured images. Variations in the illumination must be avoided in the image.

Background Estimation: A captured image is preprocessed to reduce the noise in the images. Then the image is enhanced to identify the background difference from the object.

Segmentation: Segmentation is a process that partitions the image into regions. The enhanced image is segmented and used to identify the required object for detection and tracking. The profile of the object $(\mathrm{X}, \mathrm{Y})$ with respect to coordinate position of the frame is identified. The points are plotted as a map in the continuous frames.
There are 2 ways for object map: 1) Feature Extraction and 2) Motion Analysis.

Feature Extraction: The features of the object such as shape and the edges of the object are identified.

Motion Analysis: Identify the (X, Y) position of the required object in the first frame. This process is repeated for the remaining frames. By applying affine transformation for the frames given in Fig 3, find the position change of the object, and hence the object movement can be confirmed.

Classification: Object features and the objects position information are given to the classification module.

\section{Framework of Motion analysis component}

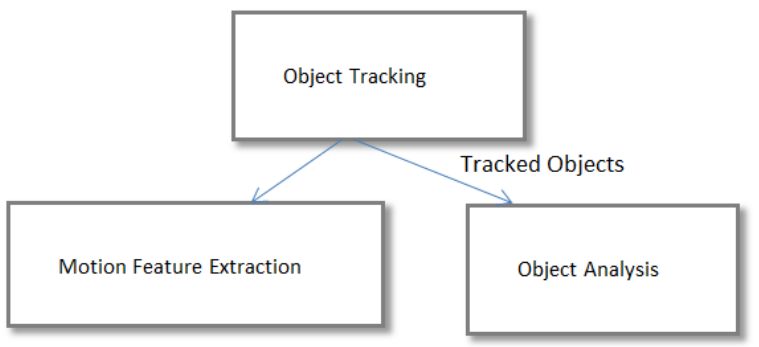

Fig. 3 : Motion analysis module

Motion analysis component consists of three major modules: Object Tracking, Motion Feature Extraction and Object Analysis. The inputs and outputs of each module and their relationships with other modules are highlighted in Figure 3. Three main outputs in the motion analysis component are:

Tracked Object: Each single rigid and moving / static object is tracked and highlighted in different colors. Each object is enclosed with rectangle, with the following properties:

i) $\quad \mathrm{X}$ and $\mathrm{Y}$ coordinates of the top left and bottom right corner of the rectangle

ii) $\quad \mathrm{X}$ and $\mathrm{Y}$ coordinates of the contour points of the tracked objects

Motion Features: The extracted features for each object are listed as follows: Change in size, Current speed, Acceleration value, Deceleration value and Change in Velocity.

Object Status and Label: Types of object status in terms of movement are determined based on the previous frames to current frame and are listed as follows: Static to Static, Static to Moving, Moving to Moving, Moving to Static, Sudden Stop, Sudden Speed.

\section{Conclusion}

This paper has presented sample frames extracted from video. The different parameters that will be calculated is presented. The potential objects are identified along with the related information static to static, static to moving, moving to moving, moving to static, sudden stop, sudden speed, occlusion with two or more separate objects, split of one object to more than one objects are recognized.

\section{References}

[1] Zielke T., Brauckmann M., and Von Seelen W., "Intensity and Edge - Based symmetry detection with an application to car - following" Image Understanding, 58,2,177-190,1993

[2] Koller D., Daniilidis K., and Nagel H.H., "Model - based Object tracking in Monocular image sequences of Road Traffic Scenec", International journal of Computer Vision,10,3,257-281,1993 
[3] G.Zhao and Y.Shinichi, Obstacle detection by vision system for autonomous vehicle, IEEE Intelligent Vehicle Symposium, pp. 31-36, 1993.

[4] N.M. Charkari, H.Mori, Visual vehicle detection and tracking based on the sign pattern, Advanced Robotics, Vol 9, No 4, pp. 367-382, 1995.

[5] M.Betke, E.Haritaglu and L.Davis, Multiple vehicle detection and tracking in hard real time, IEEE Intelligent Vehicles Symposium, pp. 351-356, 1996.

[6] M.Betke, E.Haritaglu and L.Davis, Multiple vehicle detection and tracking in hard real time, IEEE Intelligent Vehicles Symposium, pp. 351-356, 1996.

[7] N.Matthews, P.An, D.Charnley, and C.Harris, Vehicle detection and recognition in greyscale imagery, Control Engineering Practice, vol. 4, pp. 473-479, 1996.

[8] N.Friedman and S.Russell, Image segmentation in video sequences: a probabilistic approach, Proc. Thirteenth Conf. on Uncertainty in Artificial Intelligence (UAI 97), 1997.

[9] A.N.Rajagopalan, Philippe Burlina and R.Chellappa, Higher order statistical learning for vehicle detection in images, Proc. IEEE Intl. Conf. on Computer Vision, (Corfu, Greece), 1999, pp. 1204-1209.

[10] M.Betke, E.Haritaoglu and L.S.Davis, Real-time multiple vehicle detection and tracking from a moving vehicle, Machine Vision and Applications, Vol.12, pp. 69-83, 2000.

[11] M.Suwa, A stereo-based vehicle detection method under windy conditions, IEEE Intelligent Vehicle Symposium, pp. 246-249, 2000.

[12] Z.Sun, G.Bebis, and R.Miller, Improving the performance of on-road vehicle detection by combining gabor and wavelet features, The IEEE Fifth International Conference on Intelligent Transportation Systems ,September, 2002, Singapore.

[13] B.Leibe, A.Leonardis, and B.Schiele. Combined object categorization and segmentation with an implicit shape model .In ECCV'04 Works. On Stats Learning in Comp. Vision, pages 17-32, May 2004.

[14] A.N.Rajagopalan, and R.Chellappa. Vehicle detection and tracking in video. Center for Automation Research, Univerisyt of MaryLand, College Park, MD 20742 -3275 